Scientia Marina 71(3)

September 2007, 513-524, Barcelona (Spain)

ISSN: 0214-8358

\title{
Demersal and epibenthic assemblages of trawlable grounds in the northern Alboran Sea (western Mediterranean)
}

\author{
ESTHER ABAD ${ }^{1}$, IZASKUN PRECIADO $^{1}$, ALBERTO SERRANO ${ }^{1}$ and JORGE BARO ${ }^{2}$ \\ ${ }^{1}$ Centro Oceanográfico de Santander, Instituto Español de Oceanografía, Promontorio de San Martín, s/n, P.O. Box 240, \\ 39080 Santander, Spain. E-mail: esther.abad@ st.ieo.es \\ ${ }^{2}$ Centro Oceanográfico de Málaga, Instituto Español de Oceanografía, Puerto Pesquero s/n, P.O. Box 285, \\ 29640 Fuengirola, Málaga, Spain
}

\begin{abstract}
SUMMARY: The composition and abundance of megabenthic fauna caught by the commercial trawl fleet in the Alboran Sea were studied. A total of 28 hauls were carried out at depths ranging from 50 to $640 \mathrm{~m}$. As a result of a hierarchical classification analysis four assemblages were detected: (1) the outer shelf group (50-150 m), characterised by Octopus vulgaris and Cepola macrophthalma; (2) the upper slope group (151-350 m), characterised by Micromesistius poutassou, with Plesionika heterocarpus and Parapenaeus longirostris as secondary species; (3) the middle slope group (351-640 m), characterised by M. poutassou, Nephrops norvegicus and Caelorhincus caelorhincus, and (4) the small seamount Seco de los Olivos (310-360 m), characterised by M. poutassou, Helicolenus dactylopterus and Gadiculus argenteus, together with Chlorophthalmus agassizi, Stichopus regalis and Palinurus mauritanicus. The results also revealed significantly higher abundances in the Seco de los Olivos seamount, probably related to a higher food availability caused by strong localised currents and upwellings that enhanced primary production. Although depth proved to be the main structuring factor, others such as sediment type and food availability also appeared to be important. Differences between shelf and slope assemblages could be in part related to a greater dependence on benthic resources in the former and a higher use of planktonic resources in the latter.
\end{abstract}

Keywords: demersal fish, epibenthos, bathymetric distribution, discards, western Mediterranean, Alboran Sea, Seco de los Olivos seamount.

RESUMEN: AGRUPACIONES DEMERSALES Y BENTÓNICAS DE LOS FONDOS ARRASTRABLES DEL MAR DE ALBORÁN (MEDITERRÁNEO OCCIDENTAL). - Se estudia la composición y abundancia de la fauna capturada por barcos comerciales de arrastre en el mar de Alborán. Se llevaron a cabo 28 lances a profundidades comprendidas entre los 50 y los $640 \mathrm{~m}$. El análisis de clasificación jerárquica dio como resultado cuatro agrupaciones: (1) plataforma externa (50-150 m) caracterizada por las especies Octopus vulgaris y Cepola macrophthalma, (2) talud superior $(151-350 \mathrm{~m})$, caracterizada por Micromesistius poutassou, con Plesionika heterocarpus y Parapenaeus longirostris como especies secundarias, (3) talud medio (351-640 m) caracterizada por M. poutassou, Nephrops norvegicus y Caelorhincus caelorhincus, y (4) el monte submarino Seco de los Olivos (310$360 \mathrm{~m}$ ), donde M. poutassou, Helicolenus dactylopterus y Gadiculus argenteus son las especies más características, junto a otras como Chlorophthalmus agassizii, Stichopus regalis y Palinurus mauritanicus. Los resultados revelan una abundancia de megafauna significativamente mayor en Seco de los Olivos, probablemente relacionada con una mayor disponibilidad de alimento provocada por las fuertes corrientes y afloramientos que tienen lugar en la zona, y que generan un aumento en la producción primaria. Aunque la profundidad constituye el principal factor estructurante de las comunidades, también son importantes el tipo de sedimento y la disponibilidad de alimento. Las diferencias encontradas entre las comunidades de plataforma y de talud podrían estar en parte relacionadas con una mayor dependencia de los recursos bentónicos en la primera, y un mayor aprovechamiento de los recursos planctónicos en la segunda.

Palabras clave: peces demersales, epibentos, distribución batimétrica, descartes, Mediterráneo occidental, mar de Alborán, montaña submarina Seco de los Olivos. 


\section{INTRODUCTION}

The Alboran Sea is an interesting area due to its location on the border between three biogeographic marine regions, the Lusitanian, Mauritanian and Mediterranean regions, and the fact that it contains species typical of all three (Templado et al., 1986). This transition zone between the Atlantic and the western Mediterranean generates a highly peculiar and complex environment. Its hydrological characteristics are defined primarily by two main events: the Atlantic Jet, an input of surface waters through the Gibraltar strait which generates anticyclonic patterns in the current system and upwelling of organic-enriched deep-water (Heburn and LaViolette, 1990; Sarhan et al., 2000); and the Almería-Orán front, which forms at the meeting point of Atlantic and Mediterranean surface waters, at the eastern end of the Alborán Sea (Tintoré et al., 1988), representing a distribution barrier for several species and producing an enhancement of primary producers in its proximity (Fielding et al., 2001).

The Alboran Sea's geomorphology is also a source of environmental variability. The continental shelf is characterised by two types of surface: narrow areas (10 km average) with low gradient slopes, and wide areas (21 km average) with steep slopes (Flanagan, 1972; Ercilla et al., 1992). The shelf break is located around $150 \mathrm{~m}$ depth and the foot of the slope at around $1200 \mathrm{~m}$ (Hernández-Molina, 1993). The Alboran Sea is a very active region inside the area of continental collision generated by the northward movement of the African plate relative to Europe (Maldonado et al., 1992). This tectonic situation between two major plates is characterised by a complex physiographic bottom (Ballesteros et al., in press). Volcanism is expressed in seamounts like the Seco de los Olivos and the Alboran Island (Maldonado and Comas, 1992). All these factors generate a high topographical complexity, a wide range of substrates, and different types of habitats.

This hydrological and geological diversity is bound to affect the biological richness of the region, which includes a wide range of species, many of which are of commercial interest. In the western Mediterranean trawling is the most important fishery, regarding both volume of catches and economic value (Sánchez et al, 2004). Several trawl fisheries belonging to Mediterranean fishing ports of Andalucía operate all year round in the area. The most important target species are Merluccius mer- luccius, Parapenaeus longirostris, Octopus vulgaris, Nephrops norvegicus and Lophius spp. due to their high economic value, and Micromesistius poutassou and Plesionika heterocarpus because of their abundant landings.

Integrated assessment of fisheries requires studies that focus on the whole ecosystem and not only on single species, and that consider fishing activities as key pressures affecting several ecosystem components (Gaertner et al., 2005; Massuti and Reñones, 2005). Therefore, it is highly necessary to develop studies like these, which identify the components, assemblage structure and functioning of ecosystems at a regional scale.

Furthermore, the presence of sensitive and vulnerable habitats, such as seamounts and volcanic crests, has resulted in a recent proposal for making the seamounts and volcanic cones of Alboran Sea a Marine Protected Area (WWF/Adena, 2006). The Texel-Faial criteria for the identification of species and habitats that need protection, defined by OSPAR (2003), requires a detailed knowledge of the quality, structure and functioning of the ecosystems and/or habitats proposed as MPAs. Despite the ecological and economical interest of the area, there is a lack of studies on Alboran demersal and epibenthic communities, with the exception of a few studies in the western Mediterranean focusing on demersal fish (Cartes et al., 2002; Gaertner et al., 2005), elasmobranch distribution (Bertrand et al, 2000), crustaceans (Abelló et al., 2002; Cartes et al., 2002) and cephalopod assemblages (González and Sánchez, 2002) that include the Alboran Sea as one of the regions studied.

The present study is the first attempt to describe the spatial distribution patterns of demersal and megaepibenthic fauna exploited by trawlers in the Alboran Sea. The main objectives were to characterise demersal and epibenthic assemblages caught by the commercial fleet operating in the area, and to establish the relationships between demersal and epibenthic fauna distribution patterns and environmental characteristics such as depth and bottom type.

\section{MATERIAL AND METHODS}

\section{Sampling}

The study area included the north Alboran continental shelf and slope between $100 \mathrm{~m}$ and $650 \mathrm{~m}$ depth, stratified in 5 depth strata according to tradi- 


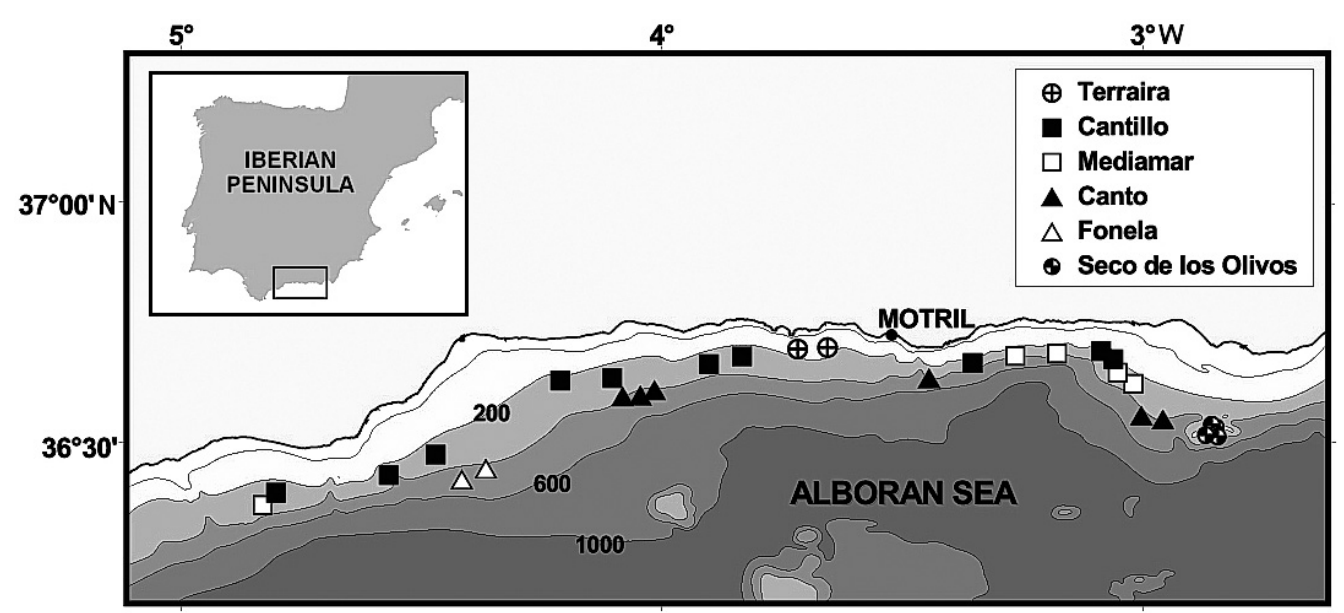

FIG. 1. - Map of the study area (north Alboran Sea, western Mediterranean Sea) showing the location of the 28 hauls in the 6 fishing grounds.

tional fishing grounds: Terraira (50-150 m), Cantillo (151-275 m), Mediamar (276-350 m), Canto (351$460 \mathrm{~m}$ ) and Fonela (461-640 m) (Fig. 1). Additional sampling was carried out on the soft bottoms of the summit of Seco de los Olivos (310-360 m), also known as Chella Bank, which is one of the small seamounts of the Alboran Sea and has an uneven topography of coarse sands and rocky bottoms.

In this area bottom trawling is performed 5 days a week for a maximum of 12 hours per day during daylight. Data were collected from a total of 28 hauls onboard eight commercial vessels representative of the trawlers constituting the trawl fleet of Motril, from August 2000 to June 2001. This study comes from a project in which data were obtained onboard commercial vessels that did not modify their usual behaviour, fishing when and where the skipper decided. The number of hauls in a fishing ground was determined by the frequency vessels went to that area in that period: 2 hauls in Terraira, 10 in Cantillo, 5 in Mediamar, 6 in Canto, 2 in Fonela and 3 in Seco de los Olivos (Fig. 1). A 44/60 otter trawl net was used, with slight inter-vessel variations (mean mesh size $=42.3 \mathrm{~mm}, \mathrm{SD}=1.6$ ). Average values of technical characteristics were: $40.1 \mathrm{TRB}$ $(\mathrm{SD}=4.9), 19.8 \mathrm{~m}$ in length $(\mathrm{SD}=1.2)$ and $219.3 \mathrm{CV}$ of power ( $\mathrm{SD}=35.6$ ), again showing slight inter-vessel variations. Therefore, the vessels were very similar and did not show important differences in technical characteristics. Hauls lasted between 2 and 6 hours, so abundance data were standardised to $\mathrm{kg} \cdot \mathrm{h}^{-1}$. Haul duration was determined by depth, since short hauls corresponded to shallower grounds and longer ones to deeper grounds. The geographical position of each haul was recorded using GPS (Global Position System). Total catch was estimated per haul. Wet weight of each commercial species was obtained from each haul. Discarded species composition was estimated on a sub-sample depending on the quantity of discarded catches and extrapolated to the total discards (Sánchez et al., 2004). This was necessary in order to identify all species caught by trawlers, because most of them are always discarded. It should be noted that the otter trawl collected only demersal and epibenthic species (Massutí and Reñones, 2005). No endobenthic species were used in the statistical analysis.

Sedimentary characteristics were obtained from Ballesteros et al. (in press), as a qualitative scale of mean particle diameter $\left(\mathrm{Q}_{50}\right)$ in coarse sands $(>500$ $\mu \mathrm{m})$, fine sands $(62-500 \mu \mathrm{m})$ and mud $(<62 \mu \mathrm{m})$. Three types of sediment were defined in the area, ranging from those with a maximum percentage of mud to those with high gravel content. The continental shelf area fishing ground (Terraira) was characterised mainly by fine sands, the slope bottoms by mud with a low sand content and the trawlable areas of the Seco de los Olivos seamount by coarse sands (Ballesteros et al., in press).

\section{Spatial distribution patterns of demersal and epibenthic fauna}

Demersal and epibenthic species abundance data were fourth-root transformed and subjected to cluster analysis using the Bray-Curtis similarity index. Prior to the cluster analysis, species with pelagic behaviour (e.g. Scomber scombrus, Boops boops, Alloteuthis spp., myctophids and Pasiphaea sivado) as well as endobenthic species (e.g. Spatangus purpureus and Sternaspis scutata) were removed. The distance matrix was then processed using the 
UPGMA algorithm. SIMPER analyses were also run to identify the species responsible for the major similarities in the dendrogram.

Once cluster groups had been established, demersal and epibenthic fauna abundance (average wet weight $h^{-1}$ ), species richness (average species number per haul) and Shannon-Wiener diversity (species wet weight per haul) for each cluster group were calculated. Differences in faunal abundance, species richness and species diversity among cluster groups were examined using a 1-way ANOVA test and Kruskal-Wallis 1-way ANOVA on ranks for homocedastic and heterocedastic data, respectively. When significant differences were detected, we ran pairwise "a posteriori" Student-Newman-Keuls and Dunn tests, respectively, to identify the groups responsible for such differences.

\section{Effect of environmental variables on demersal and epibenthic fauna distribution}

To assess the amount of variation in demersal and epibenthic fauna abundance per haul related to 3 environmental variables (i.e. depth, geographical location and sedimentary characteristics), we used a canonical correspondence analysis (CCA). Depth was considered as a continuous variable recorded as depth of each haul. Geographical location was recorded as geographical longitude of each haul and sedimentary characteristics as a qualitative variable: mud (1), fine sand (2) and coarse sand (3). CCA calculations were based on log-transformed abundance of all species collected and down-weighting of rare species. The statistical significance of the first and all canonical axes together was tested with MonteCarlo tests using 999 permutations under the reduced model. CCA results were presented graphically in a bi-dimensional ordination diagram generated by bi-plot scaling focusing on inter-species distances, in which species are represented by points and environmental variables by vectors.

\section{RESULTS}

\section{Faunal composition}

A total of 143 species were identified from the catch of the trawler fleet. Most of the species belonged to fish (67), followed by crustaceans (39), molluscs (24), echinoderms (9), annelids (2), and cnidarians (2). Blue whiting (Micromesistius poutassou) was the most abundant species in 4 of the 6 fishing grounds (Table 1), followed by the crustaceans Liocarcinus depurator and Solenocera membranacea in Mediamar (276-350 m), Plesionika heterocarpus and Parapenaeus longirostris in Cantillo (151-275) and Helicolenus dactylopterus and Gadiculus argenteus in Seco de los Olivos (310$360)$. In the Terraira fishing ground $(50-150 \mathrm{~m})$ the dominant species were Boops boops and Octopus vulgaris, and Galeus melastomus, Nephrops norvegicus and Merluccius merluccius in Fonela (461-640 m). In the Canto fishing ground (351-460 $\mathrm{m})$, M. poutassou and G. melastomus were the most abundant fish species and the commercial crustaceans species $N$. norvegicus and Plesionika martia were also very abundant. Seco de los Olivos showed the highest total catch $\left(119.01 \mathrm{~kg} \mathrm{~h}^{-1}, \mathrm{SD}=2.58\right)$, followed by Fonela $\left(58.29 \mathrm{~kg} \mathrm{~h}^{-1}, \mathrm{SD}=1.35\right)$, Terraira (53.60 kg h-1, SD = 1.51), Cantillo (49.56 kg $\left.\mathrm{h}^{-1}, \mathrm{SD}=0.51\right)$, Mediamar $\left(40.20 \mathrm{~kg} \mathrm{~h}^{-1}, \mathrm{SD}=0.70\right)$ and Canto $\left(28.53 \mathrm{~kg} \mathrm{~h}^{-1}, \mathrm{SD}=0.26\right)$.

\section{Demersal and epibenthic fauna assemblages}

The cluster analysis showed a depth-dependent grouping pattern (Fig. 2). Four groups appeared at a similarity level of 40\%: (1) the Outer Shelf (OS), corresponding to the Terraira fishing ground (100$150 \mathrm{~m}$ ); (2) the Upper Slope (US), corresponding to the Cantillo and Mediamar fishing grounds (151$350 \mathrm{~m}$ ); (3) the Middle Slope (MS), corresponding to the deepest hauls located in the Canto and Fonela fishing grounds (351-640 m); and (4) the Seco de los Olivos seamount (SS) at 310-360 m depth. The first dichotomy (28\%) showed group OS as the most different of the four groups, when shelf hauls were separated from slope hauls. The SIMPER analysis showed that the OS group was characterised by $O$. vulgaris, Cepola macrophthalma, Lophius spp. and Illex coindetti. These species, together with Pagellus acarne, were responsible for dissimilarities between the other three cluster groups (Table 2). The upper slope hauls (US) were characterised by $M$. poutassou, Plesionika heterocarpus, Parapenaeus longirostris and Gadiculus argenteus, whereas the middle slope hauls (MS) were typified by M.poutassou, Nephrops norvegicus, Caelorhincus caelorhincus and Phycis blennoides. The SS group was split in a second dichotomy out of the bathymetric pattern of clustering, showing its peculiarity with respect to 
TABLE 1. - Total catch $\left(\mathrm{kg} \cdot \mathrm{h}^{-1} \pm \mathrm{SD}\right)$ of all species sampled in the 6 fishing grounds: Terraira (50-150 m), Cantillo (151-275 m), Mediamar $(276-350 \mathrm{~m})$, Canto $(351-460 \mathrm{~m})$, Fonela $(461-640 \mathrm{~m})$ and Seco de los Olivos $(310-360 \mathrm{~m})$. Species codes are given in the first column (species with no code were not taken into account in the statistical analysis due to their pelagic or endobenthic behaviour). Asterisks mean values lower than 0.01 .

\begin{tabular}{|c|c|c|c|c|c|c|c|}
\hline \multirow[b]{2}{*}{ Code } & \multirow{2}{*}{$\begin{array}{l}\text { Assemblages } \\
\text { Fishing grounds }\end{array}$} & \multirow{2}{*}{$\begin{array}{l}\text { Outer Shelf } \\
\text { Terraira }\end{array}$} & \multicolumn{2}{|c|}{ Upper Slope } & \multicolumn{2}{|c|}{ Middle Slope } & \multirow{2}{*}{$\begin{array}{c}\text { Seco Seamount } \\
\text { Seco Olivos }\end{array}$} \\
\hline & & & Cantillo & Mediamar & Canto & Fonela & \\
\hline \multicolumn{8}{|c|}{ FISHES } \\
\hline Aimp & Arnoglossus imperialis & $0.34 \pm 0.06$ & - & - & - & - & - \\
\hline Arue & Arnoglossus rueppelii & $0.72 \pm 0.09$ & $0.05 \pm 0.01$ & - & - & - & $0.86 \pm 0.29$ \\
\hline Atho & Arnoglossus thori & $0.7 \pm 0.08$ & - & - & - & - & - \\
\hline Bpro & Bathysolea profundicola & 0.03 & $0.26 \pm 0.11$ & 0.03 & $0.05 \pm 0.01$ & - & - \\
\hline \multirow[t]{2}{*}{ Boce } & Blennius ocellaris & 0.11 & - & - & - & - & - \\
\hline & Boops boops & $12.74 \pm 6.01$ & - & - & - & - & $527^{-}+07$ \\
\hline $\begin{array}{l}\text { Ccae } \\
\text { Cmac }\end{array}$ & Caelorinchus caelorinchus & $\begin{array}{c}0.06 \\
0.54+0.21\end{array}$ & $2.69 \pm 0.56$ & $\begin{array}{c}1.28 \pm 0.4 \\
0.02\end{array}$ & $2.57 \pm 0.32$ & $2.76 \pm 0.07$ & $5.27 \pm 0.7$ \\
\hline Cape & Capros aper & $0.42 \pm 0.26$ & $1.3 \pm 0.14$ & $0.56 \pm 0.14$ & 0.01 & - & $0.42 \pm 0.07$ \\
\hline Cegr & Centrophorus granulosus & - & - & - & - & 3.43 & - \\
\hline \multirow[t]{2}{*}{ Cema } & Cepola macrophthalma & $2.58 \pm 0.26$ & $0.6 \pm 0.17$ & - & - & - & - \\
\hline & Cerastocopelus maderensis & - & - & 0.02 & - & - & - \\
\hline Cmon & Chimaera monstrosa & - & - & - & $0.07 \pm 0.03$ & - & - \\
\hline Caga & Chlorophthalmus agassizi & - & - & - & - & - & $2.25 \pm 0.24$ \\
\hline Ccon & Conger conger & $0.99 \pm 0.35$ & $0.99 \pm 0.13$ & $0.57 \pm 0.06$ & $1.17 \pm 0.15$ & $4.81 \pm 0.37$ & $3.55 \pm 0.3$ \\
\hline Dlic & Dalatias licha & - & - & - & 0.18 & - & - \\
\hline Dpas & Dasyatis pastinaca & - & 0.09 & - & - & - & - \\
\hline \multirow{2}{*}{ Dqua } & Deltentosteus quadrimaculatus & 0.19 & - & - & - & - & - \\
\hline & Diaphus holti & - & - & 0.01 & - & - & - \\
\hline Eden & Epigonus denticulatus & - & $0.02 \pm *$ & $0.12 \pm 0.08$ & $0.04 \pm 0.01$ & - & 0.02 \\
\hline Espi & Etmopterus spinax & - & - & - & $0.76 \pm 0.36$ & 0.26 & - \\
\hline Garg & Gadiculus argenteus & - & $2.35 \pm 0.22$ & $2.15 \pm 0.49$ & $0.26 \pm 0.04$ & 0.02 & $11.64 \pm 1.57$ \\
\hline Gbis & Gaidropsaurus biscayensis & - & 0.01 & $0.34 \pm 0.05$ & $0.5 \pm 0.19$ & - & - \\
\hline Ggal & Galeorhinus galeus & - & - & 1.17 & - & - & - \\
\hline Gmel & Galeus melastomus & 0.07 & - & $0.15 \pm 0.02$ & $3.86 \pm 0.47$ & $12.81 \pm 1.74$ & - \\
\hline Gnig & Gobius niger & $0.27 \pm 0.16$ & - & - & - & - & - \\
\hline Hdac & Helicolenus dactylopterus & $0.59 \pm 0.11$ & $1.1 \pm 0.14$ & $0.57 \pm 0.11$ & $0.18 \pm 0.02$ & $1.66 \pm 0.04$ & $20.43 \pm 2.56$ \\
\hline Hmed & Hoplostethus mediterraneus & - & 0.14 & $0.1-0.05$ & $0.86 \pm 0.12$ & $0.37 \pm 0.06$ & - \\
\hline & Lampanyctus crocodilus & - & 0.02 & - & - & 0.02 & - \\
\hline Lcau & Lepidopus caudatus & - & $2.01 \pm 0.65$ & $0.14 \pm 0.02$ & $0.32 \pm 0.03$ & - & - \\
\hline Ldie & Lepidotrigla dieuzeidei & 0.04 & - & - & - & - & - \\
\hline Lsan & Lesueurigobius sanzi & - & $0.05 \pm 0.01$ & $0.04 \pm 0.01$ & - & - & - \\
\hline Lnae & Leucoraja naevus & - & - & - & - & - & $2.06 \pm 0.85$ \\
\hline Loph & Lophius spp. & $4.56 \pm 1.82$ & $0.72 \pm 0.06$ & $0.64 \pm 0.1$ & $2.5 \pm 0.62$ & $4.14 \pm 0.1$ & $4.3 \pm 0.81$ \\
\hline Msco & Macroramphosus scolopax & 0.06 & 0.02 & 0.02 & - & - & 0.15 \\
\hline Mmer & Merluccius merluccius & $1.66 \pm 0.37$ & $1.53 \pm 0.21$ & $2.6 \pm 0.52$ & $1.19 \pm 0.27$ & $5.52 \pm 0.13$ & 0.28 \\
\hline Maze & Microchirus azevia & - & - & 0.23 & - & - & - \\
\hline Mvar & Microchirus variegatus & - & - & - & $0.34 \pm 0.15$ & - & - \\
\hline Mpou & Micromesistius poutassou & - & $14.41 \pm 1.72$ & $13.86 \pm 3.11$ & $3.9 \pm 0.36$ & $5.43 \pm 1.82$ & $36.47 \pm 7.01$ \\
\hline Mdyp & Molva macrophthalma & - & - & - & - & - & 0.04 \\
\hline Mbar & Mullus barbatus & - & 0.01 & - & - & - & - \\
\hline Msur & Mullus surmuletus & $0.23 \pm 0.01$ & - & - & - & - & $0.84 \pm 0.27$ \\
\hline & Myctophidae & - & $0.23 \pm 0.07$ & $0.03 \pm 0.01$ & - & - & - \\
\hline & Myctophum punctatum & - & - & * & - & - & - \\
\hline Naeq & Nezumia aequalis & - & - & - & $0.5 \pm 0.12$ & $0.16 \pm 0.1$ & - \\
\hline Oruf & Ophichthus rufus & - & 0.02 & 0.01 & - & - & - \\
\hline Oser & Ophisurus serpens & - & 0.06 & - & - & - & - \\
\hline Paca & Pagellus acarne & $4.49 \pm 2.89$ & 0.16 & - & - & - & $4.04 \pm 0.74$ \\
\hline Pbog & Pagellus bogaraveo & - & - & - & - & - & 0.29 \\
\hline Pcat & Peristedion cataphractum & - & $0.04 \pm 0.02$ & $0.16 \pm 0.11$ & - & - & 0.18 \\
\hline Pble & Phycis blennoides & - & $0.19 \pm 0.02$ & $0.66 \pm 0.14$ & $1.93 \pm 0.28$ & $4.81 \pm 0.37$ & $3.44 \pm 0.27$ \\
\hline Ssco & Scomber scombrus & 0.02 & - & - & - & - & - \\
\hline Selo & Scorpaena elongata & - & - & - & - & - & $2.27 \pm 0.63$ \\
\hline Snot & Scorpaena notata & 0.16 & - & 0.13 & - & - & - \\
\hline Spor & Scorpaena porcus & 0.10 & - & - & - & - & - \\
\hline Scor & Scorpaena spp. & - & - & 0.02 & - & - & - \\
\hline Scan & Scyliorhinus canicula & 0.43 & $1.46 \pm 0.26$ & $1.66 \pm 0.38$ & $1.41 \pm 0.14$ & - & - \\
\hline & Stomias boa & - & 0.01 & 0.01 & 0.01 & - & - \\
\hline Snig & Symphurus nigrescens & - & - & 0.97 & 0.01 & - & - \\
\hline Spha & Synchiropus phaeton & - & $0.11 \pm 0.05$ & - & - & - & $2.63 \pm 0.56$ \\
\hline Tmar & Torpedo marmorata & - & - & 0.04 & 0.03 & - & - \\
\hline Tnob & Torpedo nobiliana & - & 0.01 & - & - & - & - \\
\hline Tpic & Trachurus picturatus & - & - & - & - & - & 0.10 \\
\hline Ttra & Trachurus trachurus & $0.46 \pm 0.2$ & - & - & - & - & - \\
\hline Trtr & Trachyrincus scabrus & - & - & - & - & 0.04 & - \\
\hline Tlyr & Trigla lyra & - & - & - & - & - & $0.5 \pm 0.19$ \\
\hline Trig & Triglidae & 0.11 & 0.07 & - & - & - & - \\
\hline $\mathrm{Zfab}$ & Zeus faber & 0.25 & - & - & - & - & - \\
\hline
\end{tabular}


TABLE 1 (cont.). - Total catch $\left(\mathrm{kg} \cdot \mathrm{h}^{-1} \pm \mathrm{SD}\right)$ of all species sampled in the 6 fishing grounds: Terraira (50-150 m), Cantillo (151-275 m), Mediamar (276-350 m), Canto (351-460 m), Fonela (461-640 m) and Seco de los Olivos (310-360 m). Species codes are given in the first column (species with no code were not taken into account in the statistical analysis due to their pelagic or endobenthic behaviour). Asterisks mean values lower than 0.01 .

\begin{tabular}{|c|c|c|c|c|c|c|c|}
\hline \multirow[b]{2}{*}{ Code } & \multirow{2}{*}{$\begin{array}{l}\text { Assemblages } \\
\text { Fishing grounds }\end{array}$} & \multirow{2}{*}{$\begin{array}{l}\text { Outer Shelf } \\
\text { Terraira }\end{array}$} & \multicolumn{2}{|c|}{ Upper Slope } & \multicolumn{2}{|c|}{ Middle Slope } & \multirow{2}{*}{$\begin{array}{c}\text { Seco Seamount } \\
\text { Seco Olivos }\end{array}$} \\
\hline & & & Cantillo & Mediamar & Canto & Fonela & \\
\hline \multicolumn{8}{|c|}{ CRUSTACEANS } \\
\hline & Alpheus glaber & - & $0.04 \pm 0.01$ & $0.05 \pm 0.01$ & 0.01 & - & - \\
\hline Bmar & Bathynectes maravigna & - & - & - & 0.02 & 0.12 & - \\
\hline Cgra & Calappa granulata & - & $0.1 \pm 0.05$ & $0.07 \pm 0.04$ & 0.01 & - & $2.42 \pm 0.41$ \\
\hline Ccra & Chlorotocus crassicornis & - & $0.02 \pm 0.01$ & - & $*$ & - & - \\
\hline Darr & Dardanus arrosor & 0.06 & $0.16 \pm 0.04$ & $0.13 \pm 0.01$ & 0.05 & 0.02 & $0.29 \pm 0.08$ \\
\hline Grho & Goneplax rhomboides & - & - & $0.06 \pm 0.03$ & $0.03 \pm 0.01$ & 0.01 & - \\
\hline Hbar & Homola barbata & - & 0.02 & - & - & - & - \\
\hline Icom & Inachus communissimus & - & - & $*$ & - & - & - \\
\hline \multirow{2}{*}{ Ldep } & Liocarcinus depurator & $0.55 \pm 0.06$ & $1.51 \pm 0.09$ & $2.86 \pm 0.28$ & $0.01 \pm *$ & - & - \\
\hline & Lophogaster tipicus & - & $*$ & - & - & - & - \\
\hline Mtub & Macropipus tuberculatus & - & $0.24 \pm 0.1$ & - & $0.04 \pm 0.02$ & - & $1.03 \pm 0.14$ \\
\hline Mlon & Macropodia longipes & - & $0.06 \pm 0.03$ & - & $0.03 \pm 0.01$ & - & - \\
\hline Malo & Macropodia longirostris & 0.01 & 0.01 & $0.04 \pm 0.01$ & $*$ & - & - \\
\hline Mlan & Medorippe lanata & - & - & 0.02 & - & - & - \\
\hline Mcou & Monodaeus couchii & - & - & - & - & 0.06 & - \\
\hline Mint & Munida intermedia & - & - & - & 0.02 & - & - \\
\hline Miri & Munida iris & 0.06 & $0.12 \pm 0.01$ & $0.15 \pm 0.02$ & - & - & $0.14 \pm 0.08$ \\
\hline Nnor & Nephrops norvegicus & - & $0.03 \pm 0.01$ & $0.05 \pm 0.01$ & $1.99 \pm 0.21$ & $6.14 \pm 1.31$ & - \\
\hline Pala & Pagurus alatus & - & - & 0.02 & - & - & - \\
\hline Pexc & Pagurus excavatus & - & - & 0.01 & - & 0.05 & - \\
\hline Ppri & Pagurus prideaux & $0.46 \pm 0.27$ & - & - & - & - & 0.52 \\
\hline Pele & Palinurus elephas & - & 0.07 & - & - & - & - \\
\hline Pmau & Palinurus mauritanicus & - & - & - & 0.06 & - & $2.79 \pm 0.61$ \\
\hline Plon & Parapenaeus longirostris & - & $2.81 \pm 0.2$ & $1.56 \pm 0.22$ & - & - & - \\
\hline \multirow{2}{*}{ Pmac } & Parthenope macrochelos & - & 0.19 & - & - & - & $1.09 \pm 0.13$ \\
\hline & Pasiphaea sivado & - & $0.2 \pm 0.03$ & $0.47 \pm 0.14$ & $0.2 \pm 0.03$ & $0.08 \pm 0.03$ & - \\
\hline Pech & Philocheras echinulatus & - & - & - & $* \pm *$ & - & - \\
\hline Pisp & Pilumnus spinifer & - & $*$ & - & - & - & - \\
\hline Pant & Plesionika antigai & - & - & $0.04 \pm 0.01$ & - & - & - \\
\hline Pedw & Plesionika edwarsii & - & $0.57 \pm 0.14$ & - & * & - & - \\
\hline Pgig & Plesionika giglioli & - & - & * & $*$ & - & - \\
\hline Phet & Plesionika heterocarpus & $0.15 \pm 0.05$ & $4.01 \pm 0.35$ & $2.04 \pm 0.29$ & $0.09 \pm 0.02$ & - & $0.88 \pm 0.16$ \\
\hline Pmar & Plesionika martia & - & - & 0.25 & $1.51 \pm 0.28$ & $1.18 \pm 0.24$ & - \\
\hline Plac & Pontocaris lacazei & 0.01 & $* \pm *$ & $*$ & 0.02 & - & - \\
\hline Pspi & Pontophilus spinosus & - & $0.03 \pm 0.01$ & $0.18 \pm 0.12$ & 0.01 & - & - \\
\hline \multirow{2}{*}{ Pcan } & Processa canaliculata & - & $0.06 \pm 0.02$ & $0.09 \pm 0.04$ & $*$ & - & - \\
\hline & Sergestes arcticus & - & - & - & $* \pm *$ & 0.01 & - \\
\hline Smem & Solenocera membranacea & 0.01 & $0.87 \pm 0.12$ & $2.61 \pm 0.65$ & $0.56 \pm 0.12$ & $0.17 \pm 0.05$ & - \\
\hline Xpil & Xantho pilipes & - & - & 0.01 & $0.15 \pm 0.04$ & - & - \\
\hline \multicolumn{8}{|c|}{ MOLLUSCS } \\
\hline \multirow[t]{2}{*}{ Aver } & Abralia veranyi & - & 0.01 & 0.03 & - & - & - \\
\hline & Alloteuthis spp. & 0.02 & $0.08 \pm 0.04$ & 0.03 & - & - & - \\
\hline Bspo & Bathypolipus sponsalis & - & $*$ & - & $0.04 \pm 0.01$ & $1.15 \pm 0.24$ & - \\
\hline Ccas & Circomphalus casinus & - & $*$ & - & - & - & - \\
\hline Ecir & Eledone cirrhosa & $1.02 \pm 0.29$ & $0.21 \pm 0.02$ & 0.23 & 0.01 & - & - \\
\hline Emos & Eledone moschata & - & $0.03 \pm 0.01$ & 0.03 & 0.01 & - & - \\
\hline Icoi & Illex coindetti & $3.2 \pm 1.25$ & $0.13 \pm 0.02$ & $0.07 \pm 0.01$ & 0.06 & - & - \\
\hline Lvul & Loligo vulgaris & - & 0.01 & - & - & - & 0.59 \\
\hline Lcat & Lunatia catena & - & - & - & 0.02 & - & - \\
\hline Ncar & Neorosia caroli & - & $0.05 \pm 0.02$ & 0.09 & $0.43 \pm 0.1$ & 0.14 & $0.7 \pm 0.17$ \\
\hline Osal & Octopus salutii & - & 0.01 & - & - & - & - \\
\hline Ovul & Octopus vulgaris & $10.06 \pm 0.97$ & - & - & - & - & - \\
\hline Ptet & Pteroctopus tetracirrhus & - & - & - & 0.08 & - & - \\
\hline Rmin & Rondeletiola minor & - & $0.04 \pm 0.01$ & 0.02 & - & - & - \\
\hline Rmac & Rossia macrosoma & 0.01 & 0.30 & 0.01 & $0.14 \pm 0.02$ & - & - \\
\hline Sele & Sepia elegans & - & 0.02 & - & - & - & - \\
\hline Sorb & Sepia orbignyana & $1.44 \pm 0.21$ & $0.65 \pm 0.36$ & 0.01 & - & - & $0.7 \pm 0.34$ \\
\hline Sowe & Sepietta oweniana & 0.02 & $0.06 \pm 0.01$ & $0.1 \pm 0.02$ & - & - & - \\
\hline Sepi & Sepiola spp. & - & $0.05 \pm 0.02$ & - & - & - & - \\
\hline Tsag & Todarodes sagittatus & - & $0.18 \pm 0.02$ & $0.24 \pm 0.03$ & $0.26 \pm 0.06$ & $2.76 \pm 0.07$ & 0.23 \\
\hline Tebl & Todaropsis eblanae & $1.53 \pm 0.68$ & $0.1 \pm 0.06$ & $0.17 \pm 0.05$ & - & - & - \\
\hline Tsim & Turris similis & - & 0.01 & - & - & - & - \\
\hline Xcri & Хеnophora crispa & 0.01 & - & - & - & - & - \\
\hline
\end{tabular}


TABLE 1 (cont.). - Total catch $\left(\mathrm{kg} \cdot \mathrm{h}^{-1} \pm \mathrm{SD}\right.$ ) of all species sampled in the 6 fishing grounds: Terraira (50-150 m), Cantillo (151-275 m), Mediamar (276-350 m), Canto (351-460 m), Fonela (461-640 m) and Seco de los Olivos (310-360 m). Species codes are given in the first column (species with no code were not taken into account in the statistical analysis due to their pelagic or endobenthic behaviour). Asterisks mean values lower than 0.01 .

\begin{tabular}{|c|c|c|c|c|c|c|c|}
\hline \multirow[b]{2}{*}{ Code } & \multirow{2}{*}{$\begin{array}{l}\text { Assemblages } \\
\text { Fishing grounds }\end{array}$} & \multirow{2}{*}{$\begin{array}{l}\text { Outer Shelf } \\
\text { Terraira }\end{array}$} & \multicolumn{2}{|c|}{ Upper Slope } & \multicolumn{2}{|c|}{ Middle Slope } & \multirow{2}{*}{$\begin{array}{c}\text { Seco Seamount } \\
\text { Seco Olivos }\end{array}$} \\
\hline & & & Cantillo & Mediamar & Canto & Fonela & \\
\hline \multicolumn{8}{|c|}{ OTHER INVERTEBRATES } \\
\hline Apal & Alcyonum palmatum & - & * & $0.07 \pm 0.02$ & 0.01 & - & - \\
\hline Aacu & Aphrodite aculeata & 0.18 & $0.11 \pm 0.06$ & $0.06 \pm 0.04$ & - & - & - \\
\hline Aara & Astropecten aranciacus & - & 0.13 & - & - & - & - \\
\hline Aspi & Astropecten spinolosus & - & - & 0.01 & - & - & - \\
\hline Astr & Astropecten spp. & - & - & - & - & $0.18 \pm 0.01$ & - \\
\hline Ccid & Cidaris cidaris & - & - & - & - & - & $0.51 \pm 0.03$ \\
\hline Esep & Echinaster sepositus & - & - & - & - & - & 0.11 \\
\hline Eacu & Echinus acutus & 0.05 & - & * & - & - & - \\
\hline Prub & Pennatula rubra & $0.1 \pm 0.04$ & - & - & - & - & - \\
\hline \multirow[t]{3}{*}{ Pmic } & Psamechinus microtuberculatus & - & * & - & - & - & - \\
\hline & Spatangus purpureus & - & - & 0.01 & - & - & - \\
\hline & Sternapsis scutata & - & - & $*$ & - & - & - \\
\hline Sreg & Stichopus regalis & $1.76 \pm 0.88$ & - & - & - & - & $4.99 \pm 1.19$ \\
\hline
\end{tabular}

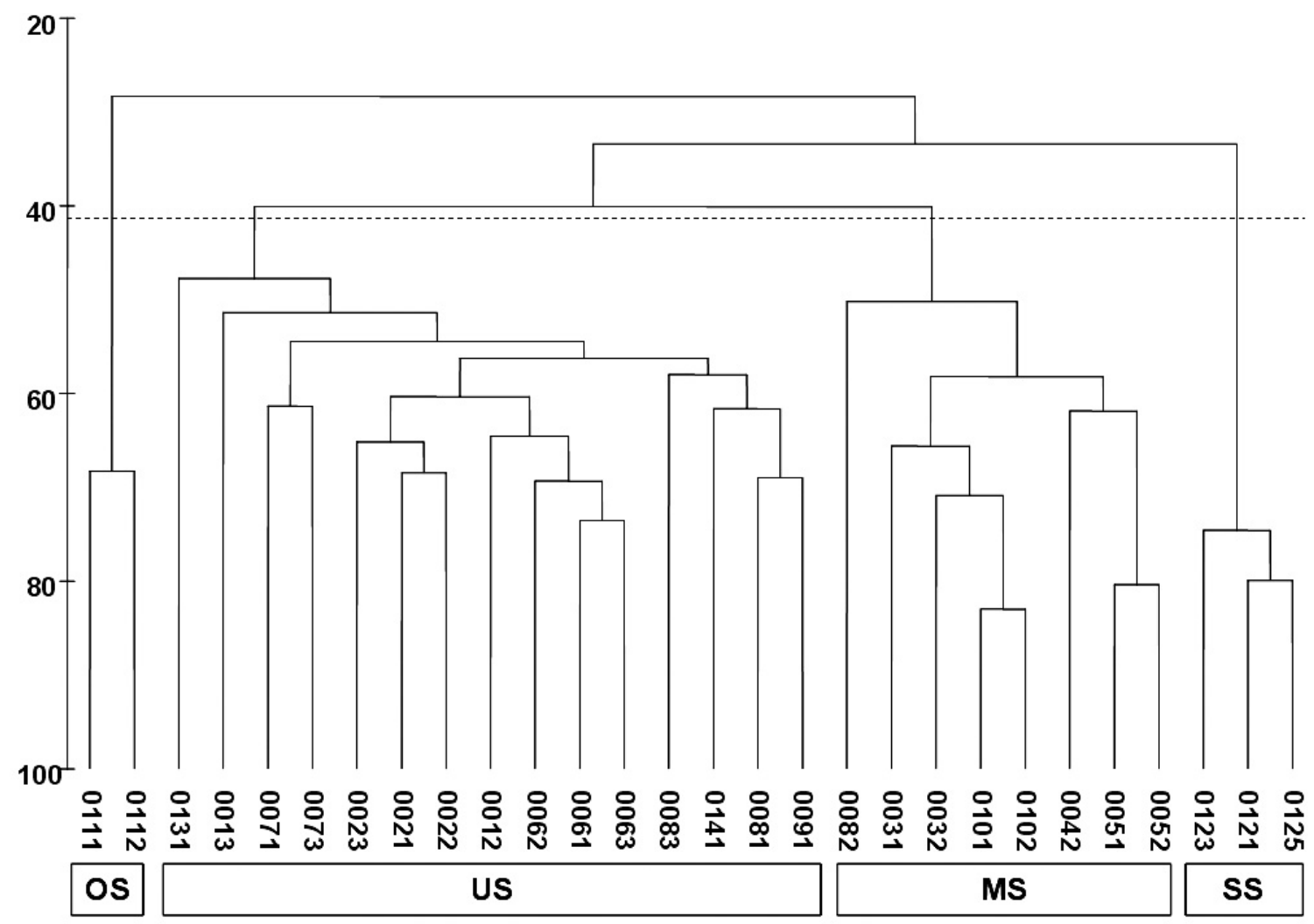

FIG. 2. - Dendrogram of hauls based on Bray-Curtis similarity of megafauna abundance. For information on species responsible for major nodes in tree topology see Table 2. Acronyms of assemblages: OS, Outer Shelf; US, Upper Slope; MS, Middle Slope; SS, Seco de los Olivos seamount.

other upper slope hauls sampled at similar depths. Although SS inter-group similarity was mainly due to common species (M. poutassou, H. dactylopterus, $G$. argenteus and $C$. caelorhincus), some scarcer species (Phycis blennoides, Chlorophthalmus agassizi, Stichopus regalis, Palinurus mauritanicus, Calappa granulata and Parthenope macrochelos) also made this fishing ground particular.
An ANOVA test and a Student-Newman-Keuls "a posteriori" test revealed that demersal and epibenthic fauna abundance was significantly higher in Seco de los Olivos (SS) than in the rest of the cluster groups $(\mathrm{F}=11.60, \mathrm{p}<0.001)$ (Fig. $3)$. However, there were no significant differences between cluster groups in either species richness or species diversity. 
TABLE 2. - Species contributing most to dissimilarity between the four groups resulting from the cluster analysis. ABU= average abundance in the cluster group; \% CUM, cumulative percentage of species contributions; OS, outer shelf; US, upper slope; MS, middle slope; SS, Seco seamount.

\begin{tabular}{|c|c|c|c|c|c|c|c|}
\hline \multicolumn{3}{|c|}{$\begin{array}{r}\text { Groups OS - US (mean dissimilarity }=68.21 \text { ) } \\
\text { ABU (OS) ABU (US) }\end{array}$} & $\%$ CUM & \multicolumn{3}{|c|}{ Groups US - MS (mean dissimilarity $=59.90$ ) } & $\%$ CUM \\
\hline Octopus vulgaris & 10.06 & 0 & 4.89 & Galeus melastomиs & 0.05 & 6.1 & 4.34 \\
\hline Micromesistius poutassou & 0 & 14.23 & 9.15 & Parapenaeus longirostris & 2.39 & 0 & 8.59 \\
\hline Pagellus acarne & 4.49 & 0.11 & 12.51 & Nephrops norvegicus & 0.04 & 3.03 & 12.56 \\
\hline Parapenaeus longirostris & 0 & 2.39 & 15.7 & Plesionika heterocarpus & 3.35 & 0.07 & 16.23 \\
\hline Gadiculus argenteus & 0 & 2.28 & 18.8 & Liocarcinus depurator & 1.96 & 0.01 & 19.87 \\
\hline Cepola macrophthalma & 2.58 & 0.4 & 21.89 & Plesionika martia & 0.08 & 1.43 & 23.05 \\
\hline \multicolumn{4}{|c|}{ Groups OS - MS (mean dissimilarity $=79.13$ ) } & \multicolumn{4}{|c|}{ Groups US - SS $($ mean dissimilarity $=65.52)$} \\
\hline & $\mathrm{ABU}(\mathrm{OS})$ & $\mathrm{ABU}(\mathrm{MS})$ & $\% \mathrm{CUM}$ & & ABU (US & $\mathrm{ABU}(\mathrm{SS})$ & $\%$ CUM \\
\hline Octopus vulgaris & 10.06 & 0 & 4.37 & Stichopus regalis & 0 & 4.99 & 3.76 \\
\hline Micromesistius poutassou & 0 & 4.28 & 7.79 & Helicolenus dactylopterus & 0.92 & 20.43 & 7.28 \\
\hline Pagellus acarne & 4.49 & 0 & 10.91 & Pagellus acarne & 0.11 & 4.04 & 10.8 \\
\hline Cepola macrophthalma & 2.58 & 0 & 14.02 & Palinurus mauritanicus & 0 & 2.79 & 14.16 \\
\hline Nephrops norvegicus & 0 & 3.03 & 17.1 & Chlorophthalmus agassizi & 0 & 2.25 & 17.4 \\
\hline Illex coindetti & 3.2 & 0.04 & 20.01 & Scorpaena elongata & 0 & 2.27 & 20.5 \\
\hline \multicolumn{4}{|c|}{ Groups OS - SS (mean dissimilarity $=68.88)$} & \multicolumn{4}{|c|}{ Groups MS - SS (mean dissimilarity $=68.57$ ) } \\
\hline & $\mathrm{ABU}(\mathrm{OS})$ & $\mathrm{ABU}(\mathrm{SS})$ & $\%$ CUM & & $\mathrm{ABU}(\mathrm{MS}$ & $\mathrm{ABU}(\mathrm{SS})$ & $\%$ CUM \\
\hline Micromesistius poutassou & 0 & 36.47 & 5.46 & Helicolenus dactylopterus & 0.55 & 20.43 & 3.75 \\
\hline Gadiculus argenteus & 0 & 11.64 & 9.67 & Stichopus regalis & 0 & 4.99 & 7.46 \\
\hline Octopus vulgaris & 10.06 & 0 & 13.77 & Gadiculus argenteus & 0.2 & 11.64 & 11.15 \\
\hline Phycis blennoides & 0. & 3.44 & 16.89 & Pagellus acarne & 0 & 4.04 & 14.82 \\
\hline Illex coindetti & 3.2 & 0 & 19.85 & Galeus melastomus & 6.1 & 0 & 18.26 \\
\hline Cepola macrophthalma & 2.58 & 0 & 22.77 & Nephrops norvegicus & 3.03 & 0 & 21.55 \\
\hline
\end{tabular}
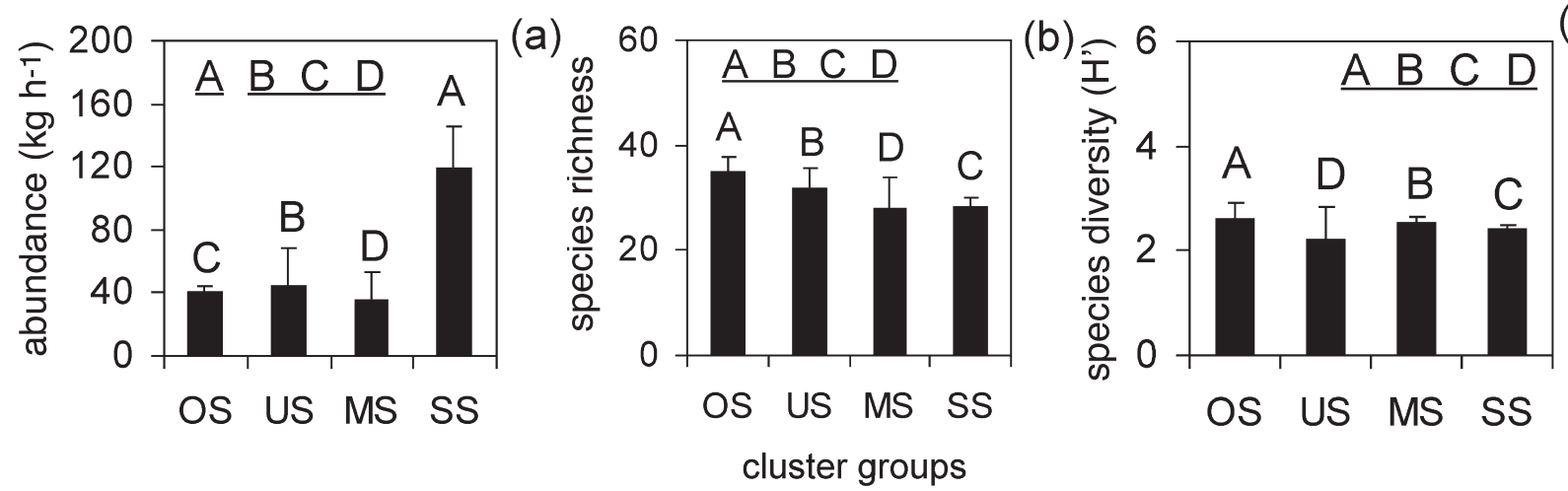

(c)

FIG. 3. - (a) Megabenthic fauna abundance $\left(\mathrm{kg} \cdot \mathrm{h}^{-1}\right)$, (b) species richness, and (c) diversity $\left(\mathrm{H}^{\prime}\right)$ per cluster group. Bars represent mean $( \pm \mathrm{SD})$ values. Uppercase letters (A to D) refer to mean values arranged in decreasing order. Groups of underlined letters indicate non-significant differences between pairs of means according to "a posteriori” Dunn's tests.

\section{Effects of depth, geographical location and sedimentary characteristics on species distribution}

The first three axes of CCA explained 30\% of the faunal variation in the species per sample matrix, and $100 \%$ of the variation in the "speciesenvironment" matrix. Montecarlo tests indicated that both the first axis $(\mathrm{p}=0.002)$ and all canonical axes together $(\mathrm{p}=0.002)$ were significant. Therefore, the environmental variables under study are clearly responsible for at least some of the faunal variation. In the CCA, both depth and bottom type $\left(\mathrm{Q}_{50}\right)$ showed a comparable weight in building axes 1 and 2 (Fig. 4a). The Longitude factor was correlated with the $\mathrm{Q}_{50}$ factor, although with less importance. Nevertheless, in the bi-plots (Fig. 4a, Fig. 4b) the high dispersion of samples and species followed a bathymetric diagonal axis. The relevance of the $\mathrm{Q}_{50}$ factor and, to a lesser extent, of Longitude, was due to the singularity of the SS group, corresponding to the Seco de los Olivos fishing ground. Coarse and medium sand covered this ground, a bottom type different from the rest. Likewise, the Terraira fishing ground (OS group) had a different bottom type consisting of fine sand, 

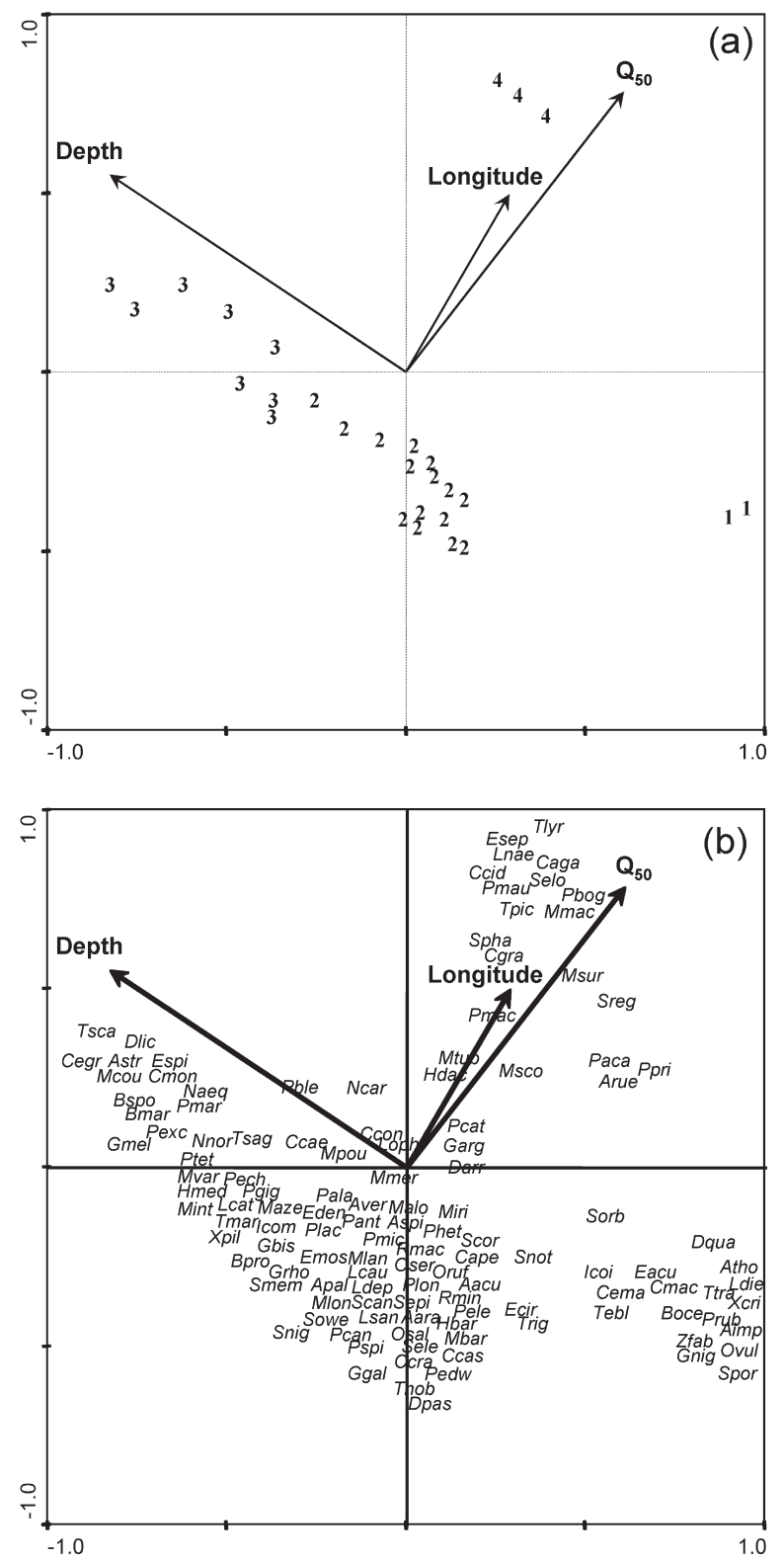

FIG. 4. - Canonical correspondence analysis (CCA) showing the bidimensional ordination of (a) hauls and (b) species. Superimposed vectors represent the environmental variables. Cluster groups: 1 , Outer Shelf; 2, Upper Slope; 3, Middle Slope; 4, Seco de los Olivos seamount. Species codes are given in Table 1.

and was the shallowest ground in the study area. The remainder of the hauls, corresponding to the slope groups (US and MS) and containing muddy bottoms, followed a bathymetric gradient.

The same response was observed in the ordination of species (Fig. 4b), again following a diagonal bathymetric gradient. The greatest discrimination was due to species related to the OS and SS groups. The outer shelf assemblage (OS group) was characterised by fish like the spotted dragonet Callyonimus maculatus, the scaldfishes Arnoglossus imperialis and A. thori, gobids such as Deltentosteus quadrimaculatus and Gobius niger, John Dory Zeus faber, horse mackerel Trachurus trachurus, the triglid Lepidotrigla dieuzeidei, and invertebrates like the mollusc Octopus vulgaris, the urchin Echinus acutus and the anthozoan Pennatula rubra. Several species were found in this habitat but also in the deeper muddy bottoms of the upper slope (US group), such as the red bandfish Cepola macrophthalma, the squids Ilex coindetti and Todaropsis eblanae, and the cuttlefish Sepia orbignyana.

Species typifying slope muddy bottoms (US and MS groups) constituted a unique group, dispersed following the bathymetric axis. Several species had affinity for shallower habitats, and were located around the centroid. These included fish (Capros aper, Ophichthus rufus, Lesuerigobius sanzi, Scorpaena notata), crustaceans (Parapenaeus longirostris, Plesionika heterocarpus, P. edwardsi, Liocarcinus depurator, Macropodia longirostris, Munida iris), molluscs (Sepia elegans, Sepiola sp., Sepietta oweniana, Rondeletiola minor), the anthozoan Alcyonum palmatum, and the polychaete Aphrodite aculeata, among others. Maximum negative values on axis 1 were for species with deeper affinities related to the MS group: the macrourids Trachyrincus scabrus, Nezumia aequalis, the slimehead Hoplostethus mediterraneus, the deep water sharks Galeus melastomus, Etmopterus spinax and Centrophorus granulosus, the rabbit fish Chimaera monstrosa, the crabs Bathynectes maravigna and Monodaeus couchii, the hermit crab Pagurus excavatus, and the octopuses Bathypolipus sponsalis and Pteroctopus tetracirrhus.

Axis 2, which highly correlated with the $\mathrm{Q}_{50}$ factor, displayed a high species dispersion of the SS group with several characteristic species, such as the fish Trachurus picturatus, Pagellus bogaraveo, Trigla lyra, Chlorophthalmus agassizi, Helicolenus dactylopterus, Synchiropus phaeton, Molva macrophthalma, Raja naevus and Scorpaena elongata, the crustaceans Palinurus mauritanicus and Calappa granulata, the squid Loligo vulgaris, the urchin Cidaris cidaris and the seastar Echinaster sepositus.

Finally, the top predators Lophius spp., Conger conger and Merluccius merluccius were located near the centroid of the analysis, since these three species were caught in all six fishing grounds (see Table 1), showing their ubiquitous distribution in the study area. 


\section{DISCUSSION}

Andalusian fishermen defined the Alboran fishing grounds as bathymetric strata with boundaries at $150,275,350$ and $460 \mathrm{~m}$. Only the small "Secos" seamounts differed from this bathymetric pattern. Our results were consistent with this definition, and confirmed depth as the first structuring factor, revealing three main assemblages, with boundaries at 150 and $350 \mathrm{~m}$. This faunal zonation has also been found in other Mediterranean Sea areas (Abelló et al., 1988; Ungaro et al, 1999; Gaertner et al., 2005). The first assemblage corresponded to the outer shelf, since the shelf break is located at $150 \mathrm{~m}$ depth (Hernández-Molina, 1993). The other two assemblages were located on the slope and have been identified as upper and middle slope. Depth also represents the main structuring factor in other areas of the Mediterranean Sea (Abelló et al., 1988; Demestre et al., 2000; Kallianiotis et al., 2000; D’Onghia et al., 2004; Gaertner et al., 2005; Massutí and Reñones, 2005), although it has often been argued that marine organisms may respond to a combination of depthrelated factors such as food availability, light, temperature and pressure (Cartes et al., 2004a).

In addition to being the shelf break depth, $150 \mathrm{~m}$ is also a hydrographical boundary, since the limit of the surface Atlantic Jet and the deeper Mediterranean waters is located approximately at this depth (Fielding et al., 2001). Furthermore, a high thermal and saline stability of the water mass below $150 \mathrm{~m}$ has been described for the whole Mediterranean Sea (Hopkins, 1985), determining the eurybathic distribution of most deepMediterranean species (Cartes et al., 2004b). Regarding the second boundary (at $350 \mathrm{~m}$ depth), between upper and middle slope, a faunal renewal belt around $300 \mathrm{~m}$ for megafaunal assemblages has been described (Cartes et al., 2004b).

The fourth assemblage corresponded to the small seamount Seco de los Olivos. The environment of this fishing ground was clearly different since rocky bottoms, inhabited by a great variety of corals and gorgonians, were interpolated with (trawlable) sandy bottoms. Therefore, the areas available to trawlers, characterised by coarse sands, were very limited and influenced by the near rocky bottoms. Similar geomorphologic formations in the study area, which are commonly known as 'Secos', have traditionally been fishing grounds. All of them have volcanic origin and can be considered as seamounts and special habitats (Maldonado and Comas, 1992; Ballesteros et al., in press). Recently, the scientific community has paid particular attention to these habitats (OSPAR, 2003) and proposed them as protected areas, although many studies are still needed to demonstrate their special characteristics and vulnerability. The Seco de los Olivos seamount showed significantly higher faunal abundances than the rest of the fishing grounds. This may indicate high food availability at this site due to strong localised currents and upwellings, which may enhance primary production and plankton biomass, resulting in alteration of the whole trophic web (Rogers, 1994; Koslow, 1997). Indeed, the planktophagous $M$. poutassou, as well as other low trophic level fish such as Gadiculus argenteus, Macrorhamphosus scolopax and Trachurus picturatus, displayed a higher abundance on the Seco de los Olivos seamount.

In addition to the bathymetric factor, the ordination analysis identified sediment type as another key factor structuring faunal assemblages in the study area. Nevertheless, all slope fishing grounds had the same bottom type, muddy sands, and were grouped into two different assemblages, upper and middle slope. The faunal renewal boundary of $350 \mathrm{~m}$ (Cartes et al., 2004b) generates a degree of variability higher than sediment type. The importance of sediment diameter in the canonical correspondence analysis was because the two most different assemblages also corresponded to different sediment types, i.e. fine sands on the shelf and coarse sands on the Seco de los Olivos seamount. Other authors have mentioned that particle size distributions alone may not always be the best guide for predicting epibenthic and demersal community types and that other factors, such as seabed morphology, appear to have a greater influence (Duineveld et al., 1991). However, to clarify the role of the sediment in structuring assemblages, a more intensive sampling would be required.

Regarding faunal composition, top predators like Lophius spp., M. merluccius and C. conger were dominant in all assemblages. The outer shelf group was characterised by typical shelf species, such as Octopus vulgaris, Arnoglossus spp., Callyonimus maculatus, Pagellus acarne and Cepola macrophthalma (Ungaro et al., 1999; Gaertner et al., 2002; González and Sánchez, 2002; Kallianiotis et al. 2004), most of them of benthophagous feeding habits. Cartes et al. (2002) described shelf fishes and 
crustaceans as more dependent on benthic resources, while plankton resources are utilised more by slope species. This pattern is a consequence of the decrease in benthic biomass with depth and the higher density of zooplankton offshore at the shelf break (Cartes et al., 2002). Slope groups, including the Seco de los Olivos seamount, were dominated by $M$. poutassou, which is a key trophic species that represents a link between nekton and demersal communities. In the upper slope group, the dominant species were the fish Caelorhincus caelorhincus and G. argenteus, and the crustaceans Plesionika heterocarpus and Parapenaeus longirostris (Abelló et al., 1988; Gaertner et al., 2005). This pandalid dominance again indicates a planktonic origin of the trophic resources (Massutí and Reñones, 2005). Another planktophagous fish, Gadiculus argenteus, was abundant on the upper slope, but especially on the Seco de los Olivos seamount, showing a bathymetric affinity for the $150-350 \mathrm{~m}$ depth stratum (Gaertner et al., 2002). Species that dominated the middle slope assemblage were typical deep-sea species, such as Galeus melastomus, C. caelorhincus and Phycis blennoides (Gaertner et al., 2002; Massutí and Moranta, 2003; Cartes et al., 2004b; D'Onghia et al., 2004), and species with mud affinity like Nephrops norvegicus (Cartes et al., 2004b).

None of the species caught on the Seco de los Olivos seamount were seamount-aggregating fish (Morato et al., 2006), although most of them have already been described as seamount fauna: e.g. Chlorophthalmus aggassizi, Pagellus bogaraveo, Molva macrophthalma, Synchiropus phaeton and Trachurus picturatus (Shcherbachev et al., 1985; Fock et al., 2002). Some of them have been regarded as hard-bottom fauna; for example, Kallianiotis et al. (2004) grouped C. aggassizi and P. bogaraveo in an upper slope assemblage inhabiting coralliferous stones, and Palinurus mauritanicus needs the proximity of rocky habitats for refuge (Goñi and Latrouite, 2005). Other species were characteristic of this bathymetric range (H. dactylopterus, $P$. blennoides, $G$. argenteus, and $C$. caelorhincus). The absence of seamount-aggregating species (p.e. orange roughy, alfonsinos) could be related to the deeper-dwelling character of these species. Furthermore, sampling on the Seco de los Olivos seamount was carried out on sandy bottoms, whereas rocky habitats, seamount slopes and less-trawled areas are more appropriate for these species (Koslow, 1997). Fauna inhabiting seamounts has been placed by several authors at the extreme end of the vulnerability spectrum (e.g. Fock et al., 2002; Morato et al., 2006). Therefore, fisheries in such areas need to be carefully controlled, since they are characterised by a boom and bust sequence, showing signs of overexploitation within a short period after the beginning of the fishery (Koslow et al., 2000; Morato et al., 2006).

The results of the present work should be taken into account for future assessment and management of trawl fisheries in the Alboran Sea, which should be based on the whole ecosystem. An integrated approach is especially necessary in vulnerable habitats such as seamounts, which are highly sensitive to anthropogenic impacts (Fock et al., 2002; Morato et $a l .$, 2006). Therefore, all the information available on habitats like the Seco de los Olivos seamount will be highly appreciated in the assessment of fisheries in the Alboran Sea.

\section{ACKNOWLEDGEMENTS}

The authors would like to thank the owners, skippers and crews of the commercial vessels from Motril, where sampling was carried out. We are also indebted to Julio Valeiras for his general support to E.A. and his valuable comments on earlier drafts of the manuscripts. Our colleagues María Gómez, María González, Ricardo Sánchez and Luis Miguel Fernández are also thanked for their valuable scientific comments.

\section{REFERENCES}

Abelló, P., F.J. Valladares and A. Castellón. - 1988. Analysis of the structure of decapod crustacean assemblages off the Catalan coast (North-West Mediterranean). Mar. Biol., 98: 39-49.

Abelló, P., A. Carbonell and P. Torres. - 2002. Biogeography of epibenthic crustaceans on the shelf and upper slope off the Iberian Peninsula Mediterranean coasts: implications for the establishment of natural management areas. Sci. Mar., 66: 2, 183-198.

Ballesteros, M., J. Rivera, A. Muñoz, A. Muñoz-Martín, J. Acosta, A. Carbó and E. Uchupi. Alboran Basin, Southern Spain. Part II: Tectonics. Mar. Petrol. Geol. (in press).

Bertrand, J., L. Gil de Sola, C. Papakonstantinou, G. Relini and A. Souplet. - 2000. Contribution on the distribution of elasmobranchs in the Mediterranean (from the Medits surveys). Biol. Mar. Mediterr., 7: 385-399.

Cartes, J.E., P. Abello, D. Lloris, A. Carbonell, P. Torres, F. Maynou and L. Gil de Sola. - 2002. Feeding guilds of western Mediterranean demersal fish and crustaceans: analysis based on a spring survey. Sci. Mar., $66: 209-220$.

Cartes, J.E., F. Maynou, J. Moranta, E. Massuti, D. Lloris and B. Morales-Nin. - 2004a. Patterns of bathymetric distribution among deep-sea fauna at local spatial scale: comparison of mainland vs. insular areas. Prog. Oceanogr., 60: 29-45. 
Cartes, J.E., F. Maynou, F. Sardá, J.B. Company, D. Lloris and S. Tudela. - 2004b. The Mediterranean deep-sea ecosystems. An overview of their diversity, structure, functioning and anthropogenic impacts, with a proposal for their conservation. Part I. IUCN, Málaga and WWF, Rome, pp 9-38.

D’Onghia, G., C. Politou, A, Bozzano, D. Lloris, G. Rotllant, L. Sion and F. Mastrototaro. - 2004. Deep-water fish assemblages in the Mediterranean Sea. Sci. Mar., 68: 87-99.

Demestre, M., P. Sánchez and P. Abelló - 2000. Demersal fish assemblages and habitat characteristics on the continental shelf and upper slope of the north-western Mediterranean. J. Mar. Biol. Ass. U.K., 80: 981-988.

Duineveld, G.C.A., A. Künitzer, U. Niemann, P.A.W.J. Wilde and J.S. Gray. - 1991. The macrobenthos of the North Sea. Neth.J. Sea Res., 28: 53-65.

Ercilla, G., B. Alonso and J. Baraza. - 1992. Sedimentary evolution of the northwestern Alboran Sea during the Quaternary. GeoMarine Letters, 12:144-149.

Fielding, S., N. Crisp, J.T. Allen, M.C. Hartman, B. Rabe and H.S.J. Roe. - 2001. Mesoscale subduction at the Almeria-Oran front. Part 2. Biophysical interactions. J. Mar. Sys., 30: 287-304.

Flanagan, J.P. - 1972. Physiography of the western Mediterranean Sea. The Alboran Sea. In: Carter, T. G., Flanagan, J. P., Jones, C. R., Marchant, F. L., Murchison, R. R., Rebman, J. H., Sylvester, J. C., Whitney, J. C. (eds). A new bathymetric chart and physiography of the Mediterranean Sea. The Mediterranean Sea: A Natural Sedimentation Laboratory, pp 6-7.

Fock, H., F. Uiblein, F. Köster and H. Von Westernhagen. - 2002. Biodiversity and species-environment relationships of the demersal fish assemblage at the Great Meteor Seamount (subtropical NE Atlantic), sampled by different trawls. Mar. Biol., 141: 185-199.

Gaertner, J.C., J.A. Bertrand and A. Souplet. - 2002. STATIS-CoA: a methodological solution to assess the spatio-temporal organization of species assemblages of the French Mediterranean Sea. Sci. Mar., 66: 221-232.

Gaertner, J.C., J.A. Bertrand, L. Gil de Sola, J.P. Durbec, E. Ferrandis and A. Souplet. - 2005. Large spatial scale variation of demersal fish assemblage structure on the continental shelf of the NW Mediterranean Sea. Mar. Ecol. Prog. Ser. 297: 245-257.

González, M. and P. Sánchez. - 2002. Cephalopod assemblages caught by trawling along the Iberian Peninsula Mediterranean coast. Sci. Mar., 66: 199-208.

Goñi, R. and D. Latrouite. - 2005. Biology, ecology and fisheries of Palinurus spp. species of European waters: Palinurus elephas (Fabricius, 1787) and Palinurus mauritanicus (Gruvel, 1911). Cah. Biol. Mar. 46: 127-142.

Heburn, G.W. and P.E. La Violette. - 1990. Variations in the structure of the anticyclonic gyres found in the Alboran Sea. J. Geo. Res., 95: 1599-1613.

Hernández Molina, F.J. - 1993. Dinámica sedimentaria y evolución durante el Pleistoceno terminal-Holoceno del margen noroccidental del mar de Alborán. Modelo de estratigrafía secuencial de muy alta resolución en plataformas continentales. $\mathrm{Ph}$. D. thesis, Univ. Granada.

Hopkins, T.S. -1985 . Physics in the sea. In: R. Margalef (ed.). Key environments: Western Mediterranean, pp 100-125. Pergamon Press, New York.

Kallianiotis, A., P. Vidoris and G. Sylaios. - 2004. Fish species assemblages and geographical sub-areas in the North Aegean Sea, Greece. Fish. Res., 68: 171-187.

Kallianiotis, A., K. Sophronidis, P. Vidoris and A. Tselepides. 2000. Demersal fish and megafaunal assemblages on the Cretan continental shelf and slope (NE Mediterranean): seasonal variation in species density, biomass and diversity. Prog. Oceanog., 46: 429-455.

Koslow, J.A. - 1997. Seamounts and the ecology of deep-sea fisheries. Amer. Scient., 85: 168-176.

Koslow, J.A., G.W. Boehlert, J.D.M. Gordon, R.L. Haedrich, P. Lorance and N. Parin. - 2000. Continental slope and deep-sea fisheries: implications for a fragile ecosystem. ICES J. Mar. Sci., 57: 548-557.

Maldonado, A. and M.C. Comas. - 1992. Geology and geophysics of the Alboran Sea: An introduction. Geo-Marine Letters, 12: 61-65.

Maldonado, A., A.C. Campillo, A. Mauffret, B. Alonso, J. Woodside and J. Campos. - 1992. Alboran Sea Late Cenozoic tectonic and stratigraphic evolution. Geo-Marine Letters, 12: 179-186.

Massutí, E. and J. Moranta. - 2003. Demersal assemblages and depth distribution of elasmobranchs from the continental shelf and slope off the Balearic Islands (western Mediterranean). ICES J. Mar. Sci., 30: 753-766.

Massutí, E. and O. Reñones. - 2005. Demersal resource assemblages in the trawl fishing grounds off the Balearic Islands (western Mediterranean). Sci. Mar. 69: 167-181.

Morato, T., W.W.L. Cheung and T.J. Pitcher. - 2006. Vulnerability of seamount fish to fishing: fuzzy analysis of life-history attributes. J. Fish Biol., 68: 209-221.

OSPAR - 2003. Criteria for the identification of species and habitats in need of protection and their method of application (the Texel-Faial Criteria). Biodiversity Committee (BDC) 03/10/1 E, Annex 5- OSPAR Convention for the protection of the marine environment of the NE Atlantic, Dublin, 2003, $14 \mathrm{pp}$.

Rogers, A.D. - 1994. The biology of seamounts. Adv. Mar. Biol. 30: 305-350.

Sánchez, P., M. Demestre and P. Martín. - 2004. Characterisation of the discards generated by bottom trawling in the northwestern Mediterranean. Fish. Res., 67: 71-80.

Sarhan, T., J. García Lafuente, M. Vargas, J.M. Vargas and F. Plaza. - 2000. Upwelling mechanisms in the northwestern Alboran Sea. J. Mar. Sys., 23: 317-331.

Shcherbachev Yu, N., E.I. Kukuev and V.I. Shlibanov. - 1985 Composition of the benthic and demersal ichthyocenoses of the submarine mountains in the southern part of the North Atlantic Range. J. Ichthyol., 25: 110-125.

Templado, J., M. García Carrascosa, L. Baratech, R. Capaccioni, A. Juan, A. López-Ibor, R. Silvestre and C. Massó. - 1986. Estudio preliminar de la fauna asociada a los fondos coralíferos del mar de Alborán (SE de España). Bol. Inst. Esp. Oceanog., 3: 93-104.

Tintoré, J., P.E. La Violette, I. Blade and A. Cruzado. - 1988. A study of an intense density front in the eastern Alboran Sea: The Almeria-Oran Front. J. Phys. Oceanog., 18: 1384-1397.

Ungaro, N., C. Marano, R. Marsan, M. Martino, M.C. Marzano, G. Strippoli and A. Vlora. - 1999. Analysis of demersal species assemblages from trawl surveys in the South Adriatic Sea. Aquat. Liv. Res., 12: 177-185.

WWF/Adena - 2006. Listado de la propuesta de WWF/Adena de Áreas Marinas Protegidas (AMP). Internet document (www.wwf.es)

Scient. ed.: J.B. Company.

Received September 26, 2006. Accepted May 15, 2007.

Published online July 9, 2007. 